\title{
Vibration Characteristic Analysis of Polar Orthotropic Sandwich Annular Plate with Tunable MR Elastomer Treatment
}

\author{
Chia-Yi Yeh (Jia-Yi Yeh) \\ Department of Digital Design and Information Management, Chung Hwa University of Medical Technology, Taiwan
}

Copyright $\mathrm{C} 2017$ by authors, all rights reserved. Authors agree that this article remains permanently open access under the terms of the Creative Commons Attribution License 4.0 International License

\begin{abstract}
Vibration analysis of polar orthotropic sandwich annular plate with magnetorheological (MR) elastomer is considered. The effects of thickness of the MR elastomer, applied magnetic fields on the sandwich system are discussed. The characteristics of the sandwich plate system can be controlled and changed by the applied magnetic fields. The core layer of MR elastomer has significant effects on the damping behavior of the sandwich plate system according to the numerical results.
\end{abstract}

Keywords Magnetorheological, Discrete Layer Finite Element, Annular Plate

\section{Introduction}

The MR material had great potential in applications for smart material and structure in recent years. The effects of the MR materials were discovered and discussed by Rabinow [1]. The MR material had rapid change in the damping and stiffness properties with the applications of the magnetic fields and presented by Weiss et al. [2]. Shiga et al. [3] obtained the magnetoviscoelastic behaviors for composite gels. Then, Dyke et al. [4] presented the designs of MR dampers and evaluations of their potential benefits in vibration suppression in structures and systems.

The applications for the construction of smart components had been previously calculated and discussed by Yalcintas and Dai [5]. Sun et al. [6] used Hamilton's principle to obtain analytical model for sandwich beam structure with MR core layer based and calculated the numerical results with simply-supported boundary condition. Ying and $\mathrm{Ni}$ [7] calculated the micro-vibration response of a stochastically excited sandwich beam with a MR elastomer core. In recently, the dynamic characteristics analysis of MR elastomer-based sandwich beam with conductive skins for various boundary conditions was calculated and discussed by Nayak et al. [8]. After that, Rajamohan and Ramamoorthy [9] studied the dynamic characterization of non-homogeneous MR fluids based multi-layer beam

There are no works had been done to discuss the sandwich polar orthotropic annular plate system with MR damping treatment to author's knowledge. The vibration characteristics of the polar orthotropic annular plate with MR core layer are calculated by using the discrete layer finite element method in present study. The characteristics of the sandwich plate system can be controlled and changed by the applied magnetic fields. And, the MR elastomer has significant effects on the damping behavior of the sandwich plate system according to the numerical results. The present numerical results can be utilized to design some active controllable mechanical devices.

\section{Methods}

In Figure 1, the sandwich polar orthotropic annular plate with ER core layer is considered. Layer 1 is the constraining layer and assumed to be pure elastic. The MR elastomer layer is designed as layer 2 and the material properties can be changed by applying various electric fields. Layer 3 is the base annular plate and assumed to be undamped, pure elastic, and polar orthotropic. The base annular plate is designed as the inner radius $r_{i}=a$ and outer radius $r_{0}=b$. Then, the following assumptions must be mentioned first. The transverse displacements of each layer are equal and there are no slipping between the constraining-MR layer and MR-base annular plate layer. 


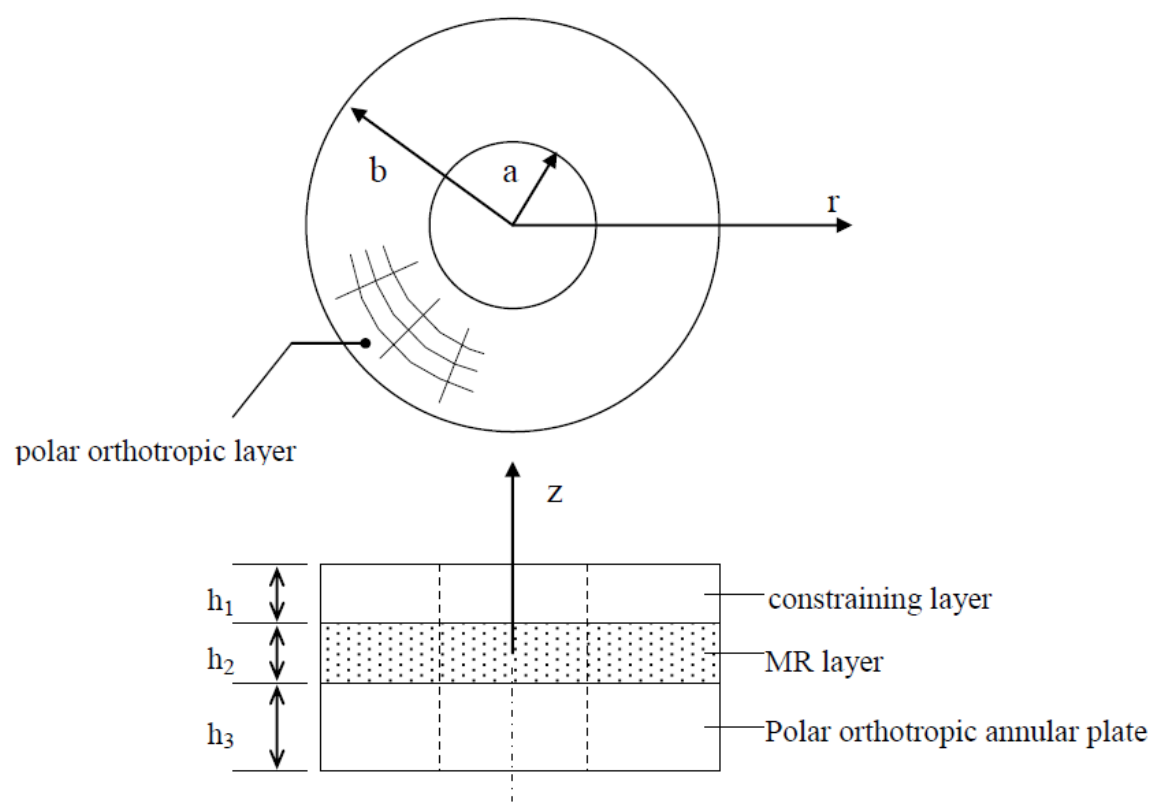

Figure 1. sandwich annular plate with MR

In order to analyze the problem, the displacement field of the layer $i$ is employed as follows:

$$
d_{i}=\left\{\begin{array}{l}
u_{i}(r, \theta, z, t) \\
w_{i}(r, \theta, z, t)
\end{array}\right\}=\left[\begin{array}{ccc}
\left(\frac{1}{2}-\frac{z}{h_{i}}\right) & \left(\frac{1}{2}+\frac{z}{h_{i}}\right) & 0 \\
0 & 0 & 1
\end{array}\right]\left\{\begin{array}{c}
U_{i}(r, \theta, t) \\
U_{i+1}(r, \theta, t) \\
W(r, \theta, t)
\end{array}\right\} .
$$

By using the interpolation in r-direction and the circumferential wave number $m$, the displacements of the interfaces for two-layer can be shown in terms of the nodal degrees of freedom as follows:

$$
\left\{\begin{array}{c}
U_{i}(r, \theta, t) \\
U_{i+1}(r, \theta, t) \\
W(r, \theta, t)
\end{array}\right\}=\left[\begin{array}{cccccccc}
\phi_{u}^{A} & 0 & 0 & 0 & \phi_{u}^{B} & 0 & 0 & 0 \\
0 & \phi_{u}^{A} & 0 & 0 & 0 & \phi_{u}^{B} & 0 & 0 \\
0 & 0 & \phi_{w}^{A} & \phi_{\Theta}^{A} & 0 & 0 & \phi_{w}^{B} & \phi_{\Theta}^{B}
\end{array}\right] q_{i}^{e}(t),
$$

where the vector of the nodal displacements of the element $q_{i}^{e}(t)=\left\{\begin{array}{llllllll}U_{i}^{A} & U_{i+1}^{A} & W^{A} & \Theta^{A} & U_{i}^{B} & U_{i+1}^{B} & W^{B} & \Theta^{B}\end{array}\right\}^{T}$, and in which $\quad \phi_{u}^{A}=(1-\xi) \cos m \theta \quad, \quad \phi_{u}^{B}=\xi \cos m \theta \quad, \quad \phi_{w}^{A}=\left(1-3 \xi^{2}+2 \xi^{3}\right) \cos m \theta \quad, \quad \phi_{w}^{B}=\left(3 \xi^{2}-2 \xi^{3}\right) \cos m \theta \quad$, $\phi_{\Theta}^{A}=\left(\xi-2 \xi^{2}+\xi^{3}\right) \cos m \theta, \phi_{\Theta}^{B}=\left(-\xi^{2}+\xi^{3}\right) \cos m \theta, \quad \xi=\frac{r-r_{i}}{r_{0}-r_{i}}$.

Then, the strain-displacement relation for the $i$ th layer of the system can be expressed as the following form:

$$
\varepsilon_{i}=\left[\begin{array}{cc}
\frac{\partial}{\partial r} & 0 \\
\frac{1}{r} & 0 \\
\frac{\partial}{\partial z} & \frac{\partial}{\partial r}
\end{array}\right] d_{i}=D d_{i},
$$

where $D$ is the differential operator matrix, and $\varepsilon_{i}=\left\{\begin{array}{lll}\varepsilon_{r, i} & \varepsilon_{\theta, i} & \gamma_{r z, i}\end{array}\right\}^{T}$.

Then, the stress-strain relation can be obtained and shown as follows:

$$
\sigma_{i}=C_{i} \varepsilon_{i},
$$

where $\sigma_{i}=\left\{\begin{array}{lll}\sigma_{r, i} & \sigma_{\theta, i} & \tau_{r \theta, i}\end{array}\right\}^{\mathrm{T}}, C_{i}$ is the elasticity matrix. 

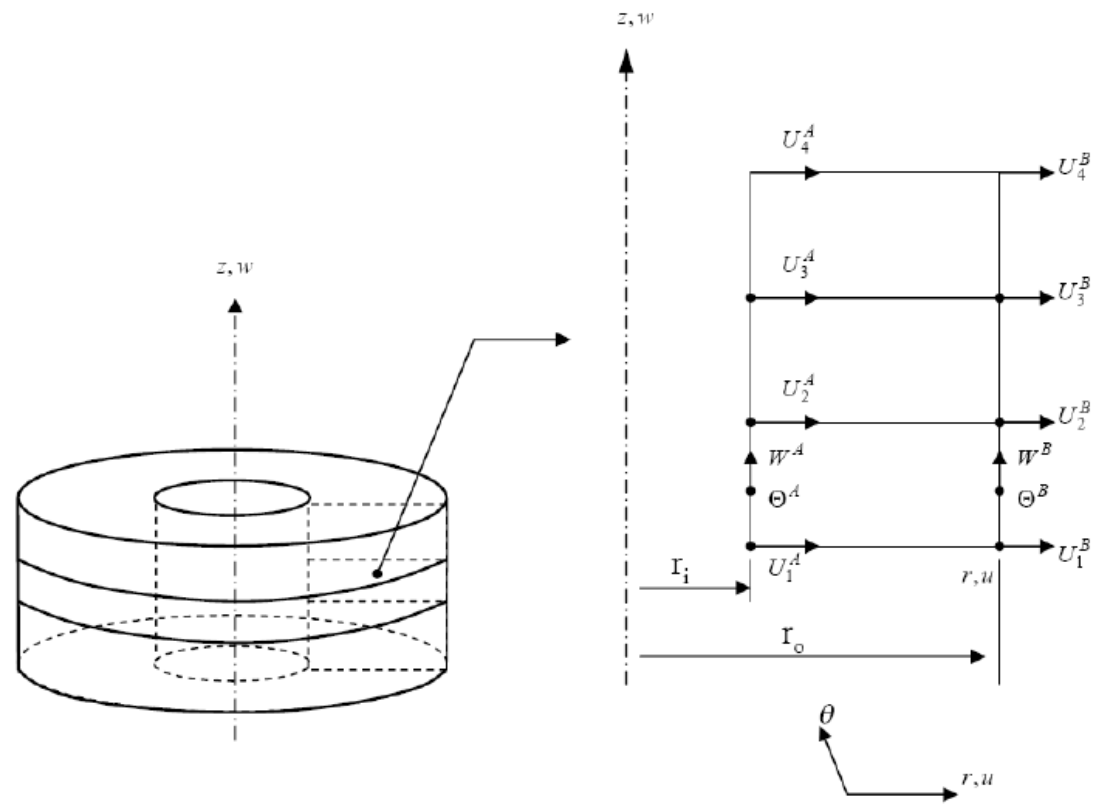

Figure 2. Discrete layer annular finite element for three-layer element.

The discrete layer finite element is utilized as shown in Figure 2. According to the above equation, the kinetic and strain energies of the element for $i$ th layer can be expressed as the following form:

$$
T_{i}^{e}=\frac{1}{2} \int_{V} \rho_{i} \dot{d}_{\mathrm{i}}^{\mathrm{T}} \dot{d}_{\mathrm{i}} d V, V_{i}^{e}=\frac{1}{2} \int_{V} \rho_{i} \sigma_{i}^{\mathrm{T}} \varepsilon_{\mathrm{i}} d V
$$

where $\rho_{i}$ is the mass density of the $i$ th layer.

The kinetic and strain energies of the element can be rewritten as follows by substituting the equation (1), (2), (3), and (4):

$$
V_{i}^{e}=\frac{1}{2} \mathrm{U}_{i}^{e^{\mathrm{T}}} \mathrm{K}_{i}^{e} \mathrm{U}_{i}^{e}, T_{i}^{e}=\frac{1}{2} \dot{\mathrm{U}}_{i}^{e^{\mathrm{T}}} \mathrm{M}_{i}^{e} \dot{\mathrm{U}}_{i}^{e}
$$

in which,

$$
\begin{gathered}
\mathrm{K}_{i}^{e}=\int_{V} \rho_{i}\left(H_{1, i} H_{2}\right)^{\mathrm{T}}\left(H_{1, i} H_{2}\right) d V, \\
\mathrm{M}_{i}^{e}=\int_{V}\left(D H_{1, i} H_{2}\right)^{\mathrm{T}} C_{i}^{\mathrm{T}}\left(D H_{1, i} H_{2}\right) d V
\end{gathered}
$$

Then, the following relations must be obtained by combining the elemental matrices into the global stiffness and mass matrices:

$$
U_{i}^{e}=\operatorname{Tr}_{i}^{e} U,
$$

where $U$ and $\operatorname{Tr}_{i}^{e}$ are the global nodal co-ordinate vector and transformation matrix, respectively.

The equation of motion for the polar orthotropic annular sandwich system can be express as the following form by assembling the contributions of all elements:

$$
M \ddot{U}+K U=0,
$$

in which,

$$
K=\sum_{i=1}^{3}\left(\sum_{e=1}^{N_{i}} T r_{i}^{e^{\mathrm{T}}} K_{i}^{e} T r_{i}^{e}\right), M=\sum_{i=1}^{3}\left(\sum_{e=1}^{N_{i}} \operatorname{Tr}_{i}^{e^{\mathrm{T}}} M_{i}^{e} T r_{i}^{e}\right)
$$

where $N_{i}$ is the element number of the $i$ th layer.

Finally, the complex eigenvalues $\tilde{\lambda}$ of the above complex eigenvalue problems can be calculated numerically. The natural frequencies $\omega$ and modal loss factor $\eta_{v}$ of the sandwich polar orthotropic annular plate with ER core layer can be obtained as follows:

$$
\omega=\sqrt{\operatorname{Re}(\tilde{\lambda})}, \eta_{v}=\frac{\operatorname{Im}(\tilde{\lambda})}{\operatorname{Re}(\tilde{\lambda})}
$$

\section{Results}

The complex shear modulus of the MR elastomer is expressed as follows [10]:

$$
G^{*}(B)=G^{\prime}(B)+j G^{\prime \prime}(B),
$$

the storage modulus

$G^{\prime}(B)=-3.3691 \times B^{2}+4997.5 \times B+873000 \quad$, the loss modulus $G^{\prime \prime}(B)=-0.9 \times B^{2}+812.4 \times B+185500$, B is the intensity of magnetic field in Gauss. Additionally, in order to simplify the following analysis and discussion, the geometric and non-dimensional parameters are used:

$v_{1}=v_{3}=0.29 \quad, \quad v_{2}=0.49 \quad, \quad \rho_{1}=\rho_{3}=2700 \mathrm{~kg} / \mathrm{m}^{3}$, $\rho_{2}=3500 \mathrm{~kg} / \mathrm{m}^{3}, E_{r, 1}=E_{r, 3}=70 \mathrm{GPa}, \kappa=\pi^{2} / 12$ (for layer 1, 3), $\kappa=1$ (for layer 2).

At first, the calculations of the natural frequencies and modal loss factors for the sandwich annular plate are listed in 
Table 1. It can be seen that the good accuracy and convergence can be found in the above comparisons. The effects of applied magnetic fields on natural frequencies and modal loss factors of the sandwich annular plate with strength parameters are presented in Figure 3(a) and (b), respectively. According to the numerical results, it can be seen that the natural frequencies increase while the applied magnetic field magnitudes increase. The modal loss factors decrease when the magnetic field strength increases. Based on the numerical results, the tendency of the natural frequency and modal loss factor is similar for strength parameters $\left(E_{\theta} / E_{r}\right) 0.5,1.0$ and 1.5.
Table 1. Comparisons between published and proposed methods for the full coverage annular plate.

\begin{tabular}{|c|c|c|c|c|}
\hline \multirow{2}{*}{$\begin{array}{l}\text { Mode } \\
(\boldsymbol{n}, \boldsymbol{m})\end{array}$} & \multicolumn{2}{|c|}{ Natural frequency $(\mathbf{H z})$} & \multicolumn{2}{c|}{ Modal loss factor } \\
\cline { 2 - 5 } & Present & Ref. [11] & Present & Ref. [11] \\
\hline$(0,0)$ & 74.44 & 74.38 & 0.1128 & 0.1127 \\
\hline$(0,1)$ & 73.00 & 73.08 & 0.09542 & 0.09576 \\
\hline$(0,2)$ & 96.20 & 96.38 & 0.1016 & 0.1021 \\
\hline$(0,3)$ & 144.0 & 142.8 & 0.1210 & 0.1212 \\
\hline$(0,4)$ & 205.2 & 203.7 & 0.1170 & 0.1177 \\
\hline
\end{tabular}

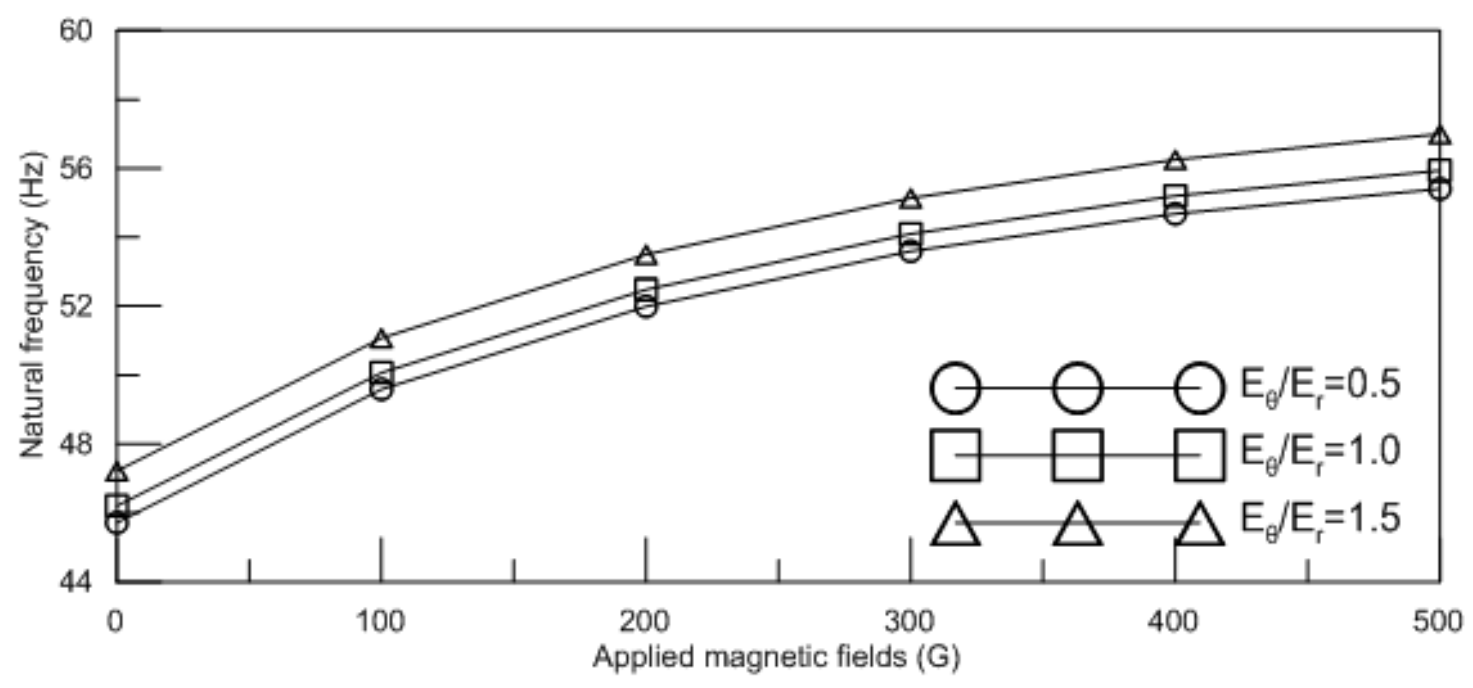

(a)

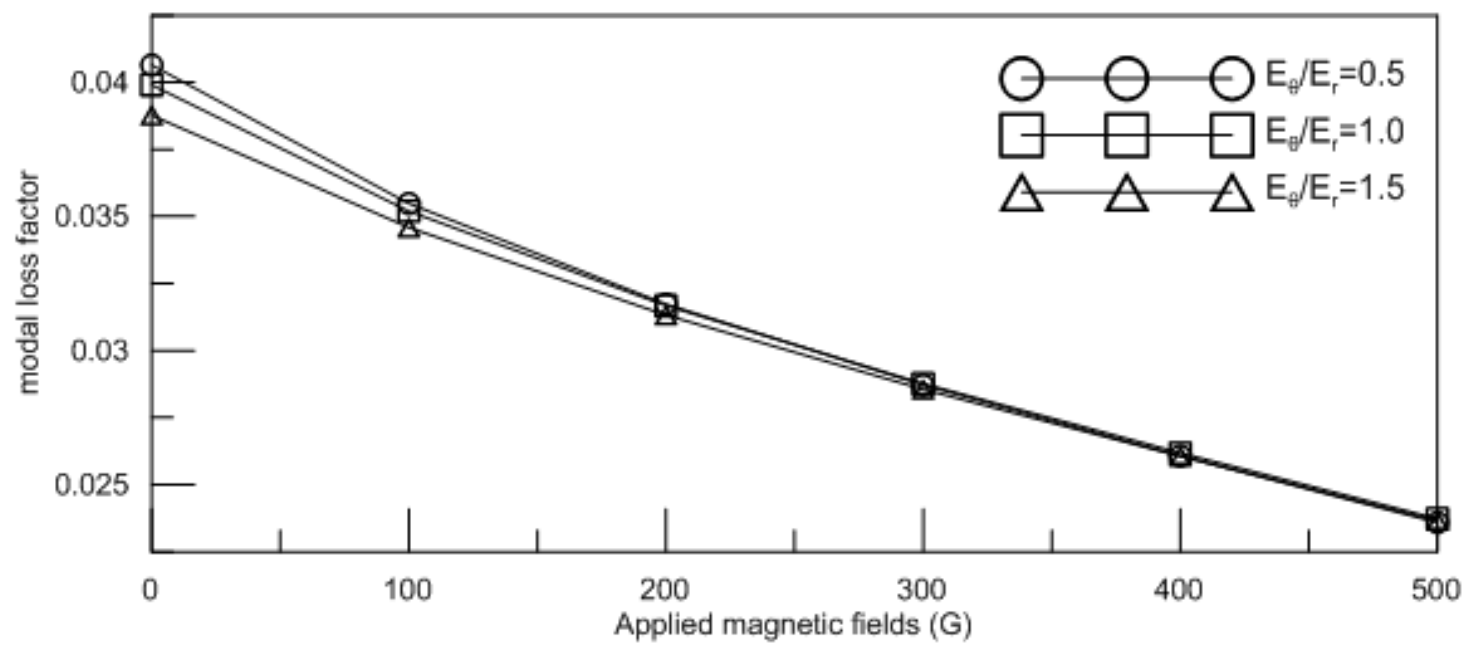

(b)

Figure 3. Variation of natural frequency and modal loss factor of the sandwich cylindrical shells with various intensity of magnetic field 


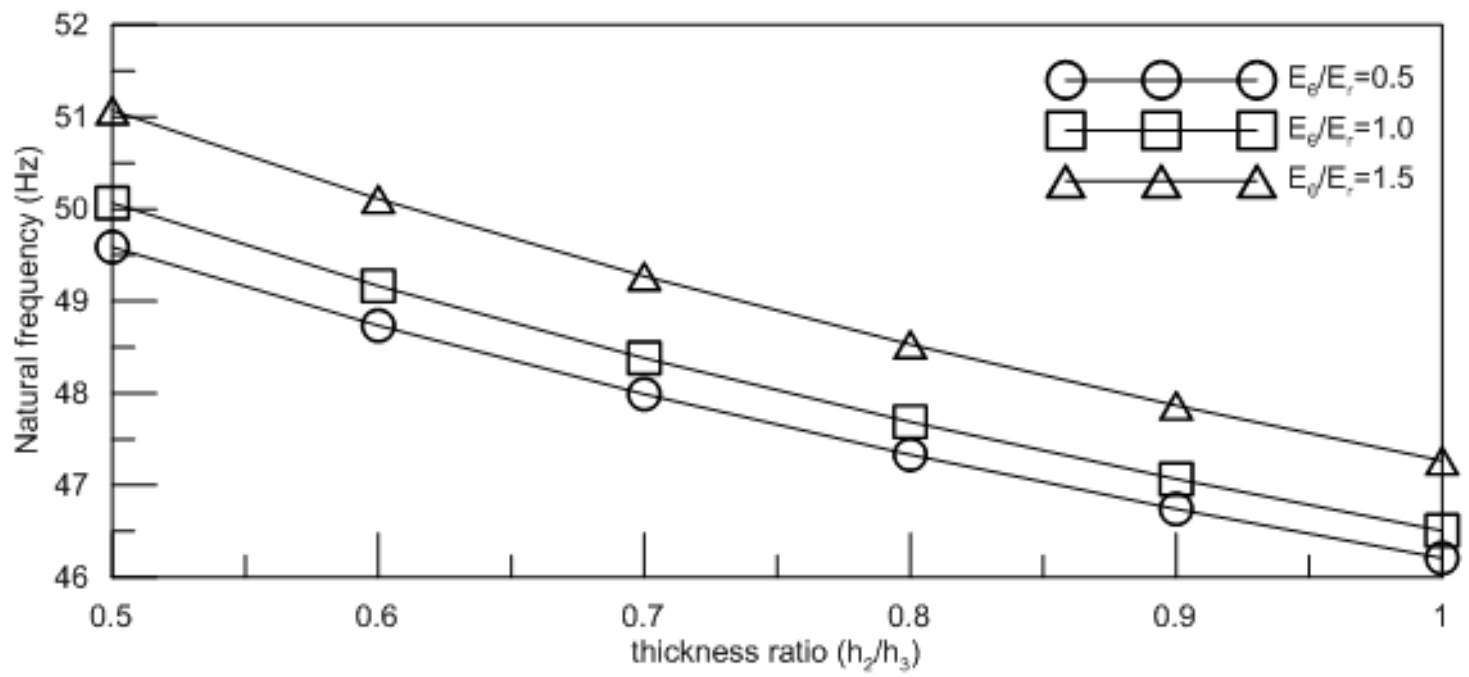

(a)

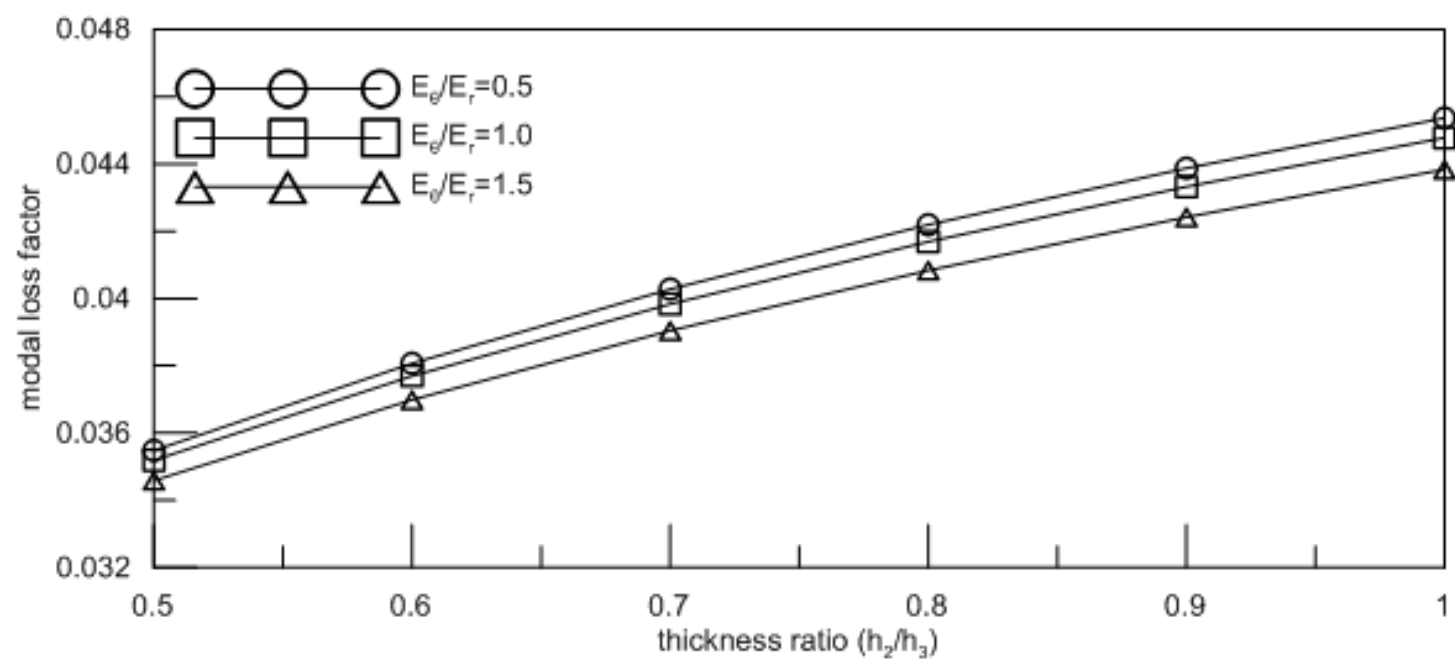

(b)

Figure 4. Variation of natural frequency and modal loss factor of the sandwich cylindrical shells with various thickness of MR layer.

Figure 4 (a) and (b) show the numerical results for the variations of sandwich annular plate of various thickness of MR layer. Based on the numerical results, it can be observed that the larger thickness of the MR layer, the smaller natural frequency. On the Contrary, the modal loss factor will be larger when the thickness of MR layer is getting larger. Besides, the natural frequency will increase and modal loss factor will decrease while strength parameter increases from the figures. Thus, the thickness of MR layer also affected the characteristics of the sandwich system on the natural frequency and modal loss factor.

\section{Conclusions}

The vibration characteristics of the sandwich polar orthotropic annular plate with MR elastomer are investigated in this study. The natural frequency and modal loss factor of the sandwich polar orthotropic annular plate system are calculated by the discrete layer finite element method. The following conclusions can be drawn based on the numerical results:

1. It can be seen that the applied magnetic fields will change the characteristics of the MR elastomer according to the results. As the applied magnetic fields increase, the natural frequencies of the sandwich annular plate system increase and the modal loss factor will decrease.

2. Additionally, it also can be observed that the larger MR elastomer thickness, the smaller natural frequency of the sandwich annular plate system. Contrary to the modal loss factor, the larger MR elastomer thickness will affect the values of the modal loss factor. 
3. The strength parameters $\left(E_{\theta} / E_{r}\right)$ can be utilized to change the vibration characteristics of the sandwich polar orthotropic annular plate system.

The applied magnetic fields and the change of the MR elastomer thickness had significant effects on the natural frequency and modal loss factor. So, we can use the characteristics to design some active, tunable and controllable mechanical devices.

\section{Acknowledgements}

This research was partially supported by the National Science Council in Taiwan through Grant MOST 105-2221-E-273-003.

\section{REFERENCES}

[1] Rabinow J. (1959). Magnetic Fluid Torque and Force Transmitting Device. U.S. Patent.

[2] Weiss K.D., Carlson J.D. \& Nixon D.A. (1994). Viscoelastic properties of magneto-and electro-rheological fluids. Journal of Intelligent Material Systems and Structures, 5, 772-75.

[3] Shiga T., Okada A. \& Kurauchi T. (1995). Magneto viscoelastic behavior of composite gels," Journal of Applied Polymer Science, 58, 787-92.
[4] Dyke S. J., Spencer B.F., Sain M. K. \& Carlson J.D. (1998). An experimental study of MR dampers on seismic protection. Smart Materials and Structures,7, 693-703.

[5] Yalcintas M. \& Dai H. (1999). Magnetorheological and electrorheological materials in adaptive structures and their performance comparison. Smart Materials and Structures, 8, 560-73.

[6] Sun Q., Zhou J.X. \& Zhang L. (2003). An adaptive beam model and dynamic characteristics of magnetorheological materials. Journal of Sound and Vibration, 261, 465-81.

[7] Ying Z.G. \& Ni Y.Q. (2009). Micro-vibration response of a stochastically excited sandwich beam with a magnetorheological elastomer core and mass. Smart Materials and Structures, 18, 095005.

[8] Nayak B., Dwivedy S. K. \& Murthy K.S.R.K. (2011). Dynamic analysis of magnetorheological elastomer-based sandwich beam with conductive skins under various boundary conditions. Journal of Sound and Vibration, 330, 1837-59.

[9] Rajamohan V. \& Ramamoorthy M. (2012). Dynamic characterization of non-homogeneous magnetorheological fluids based multi-layer beam. Applied Mechanics and Materials, 110-116, 105-112.

[10] Rajamohan V., Rakheja S. \& Sedaghati R. (2010). Vibration analysis of a partially treated multi-layer beam with magnetorheological fluid. Journal of Sound and Vibration, 329, 3451-69.

[11] Roy P.K. \& Ganesan, N. (1993). A vibration and damping analysis of circular plates with constrained damping layer treatment. Computers and Structures, 49, 269-274. 